\title{
Understanding the Platform Economy: Signals, Trust, and Social Interaction
}

\author{
Maik Hesse \\ Einstein Center Digital Future | TU Berlin \\ maik.hesse@campus.tu-berlin.de \\ David Dann \\ Karlsruhe Institute of Technology \\ david.dann@kit.edu
}

\author{
Fabian Braesemann \\ Saïd Business School | University of Oxford \\ fabian.braesemann@sbs.ox.ac.uk \\ Timm Teubner \\ Einstein Center Digital Future | TU Berlin \\ teubner@tu-berlin.de
}

\begin{abstract}
Two-sided markets are gaining increasing importance. Examples include accommodation and car sharing, resale, shared mobility, crowd work, and many more. As these businesses rely on transactions among users, central aspects to virtually all platforms are the creation and maintenance of trust. While research has considered effects of trust-building on diverse platforms in isolation, the overall platform landscape has received much less attention. However, cross-platform comparison is important since platforms vary in their degree of social interaction, which, as we demonstrate in this paper, determines the adequacy and use of different trust mechanisms. Based on actual market data, we examine the mechanisms platforms employ and how frequent users rely on them. We contrast this view against survey data on users' perceptions of the context-specific importance of these trust-building tools. Our findings provide robust evidence for our reasoning on the relation between platforms' degree of social interaction and the associated expressive trust cues.
\end{abstract}

Code and Data:

http://bit.ly/UnderstandingPlatformEconomy

\section{Introduction}

Two-sided platforms have gained accelerating importance and research attention over the last couple of years [1], [2]. Examples from the consumer-toconsumer domain include services for accommodation (e.g., Airbnb, Homestay) and carsharing (e.g., Drivy, Turo), mobility (e.g., BlaBlaCar, Uber, Wingly), ecommerce (e.g., eBay, Gumtree), crowd work (e.g., Helpling, TaskRabbit), and many more. Such platforms have caused significant changes in many incumbent businesses along with the reorganization of a wide variety of markets, work arrangements, as well as value creation and capture [3]-[5].

As platform-based business models rely on the realization of transactions among peers, a central aspect to virtually all platforms are the creation and maintenance of peer-trust [6]-[9]. While recent research has considered the effects of trust-building mechanisms on different platforms separately (e.g., how profile images and star ratings affect trust and booking intentions on Airbnb; [10]), the overall platform landscape as a whole has received much less research attention [11].

We suggest that a broader assessment of trust and reputation across platforms is urgently needed. Comprehending how platform operators act to govern and guide user behavior and usage patterns in digital platform ecosystems can allow for better understanding of resulting behaviors and outcomes - and vice versa. By offering mechanisms to build trust and reputation, platforms acknowledge that economic transactions are "socially embedded" [12]. Ratings, reviews, and expressive user profiles function as "systems of control" [13] to counter opportunism in digital environments, as they inform about the "identity and past relations of individual transactors" [12, p. 491]. Based on this reasoning, we conjecture that platforms' degree of social interaction (DoSI; i.e., the extent to which personal interactions between platform users become part of the overall value proposition and may enable positive social experiences; [14]) represents a key driver of the adequacy and use of trust cues.

Our research objective, thus, is threefold. First, based on actual market data crawled from the Internet, we provide a cross-platform overview and propose a categorization for the different mechanisms platforms employ to build and maintain trust between users. In addition to uncovering this full palette of trust-building tools available to platform users, we also highlight the frequency of utilizing said mechanisms. Second, based 
on self-reported data from a consumer survey, we analyze how the available mechanisms are actually perceived and evaluated - and thus shaped-by the specific platforms' users while also investigating which additional trust cues they would like to see. In this sense, we juxtapose the availability (platform perspective) with the usage frequency (user perspective: provider) and perceived importance (user perspective: consumer) of trust-building on two-sided markets. These perspectives help us to better understand which of the many striking observations are specific to certain platforms - and which point to more fundamental phenomena of the platform economy as a whole. Third, thanks to the multi-platform perspective presented here, we are able to compare the use and perceived importance of trust-building mechanisms with respect to a platform's degree of social interaction.

To evaluate our reasoning, we draw on two independent and unique data sets: To start with, by considering eleven platforms from various domains and assessing several characteristic statistics, including rating scores, activity concentration, and profile images from a total of over 42,000 user profiles. Next, by combining this empirical market data with quantitative and qualitative survey data that we collected from 187 participants. They evaluated the importance of different trust-building mechanisms across several platform types (e.g., accommodation, mobility, etc.).

In a nutshell, our results show that most of the investigated platforms make use of a variety of means for trust-building-yet that there exist marked differences in how users actually utilize them. Both, usage and perceived importance depend on context: the higher a platform's degree of social interaction, the more trust cues are used and deemed important.

\section{Related work}

Typical users of consumer-to-consumer (C2C) platforms are non-professional individuals with neither an established brand image nor global recognition. Consumption on these platforms requires different levels of trust [15], yet many C2C transactions yield high economic, social, and physical exposure [16]. Users of BlaBlaCar (rides) and Wingly (flights), for instance, literally put their lives into the hands of their respective driver or pilot. Trust (into the prospective good/service provider) is hence of utmost importance, as the degree of interaction between users is very high [17]. As recent research shows, high levels of trust can be achieved without prior in-person encounters [8]. We will now review which levers specifically create trust and propose a taxonomy for them.
Research from the domains of economics, information systems, and electronic commerce has studied the role of online reputation for trust-building, realization of transactions, and prices for almost two decades [7], [18], [19]. The positive economic effects of transaction-based trust cues (e.g., star ratings and text reviews; see [9] for a definition) have been broadly investigated across a range of platforms: from earlier work on realized price premiums for providers on eBay [20], [21] to more recent investigations of effects on sharing economy platforms such as Airbnb [22], [23] or BlaBlaCar [24].

In view of the ever-increasing importance of platform business models and the emergence of $\mathrm{C} 2 \mathrm{C}$ platforms with private individuals in the role of providers, online reputation and user representation are charged with particular meaning. Personal aspects and individual characteristics are essential to many $\mathrm{C} 2 \mathrm{C}$ platforms and transactions [25], and that is for at least two reasons. First, contingent on the specific platform type, social interactions between users do take place. Certainly, the extent of these encounters can varyfrom mere online communication to co-usage sharing. In the latter case, personal interactions between users are a prerequisite for the transaction to materialize. Second, scenarios that involve personal interactions may create additional, social value for consumers. This implies that providers themselves are part of the overall value proposition of the platform (pleasant and locally versed Airbnb hosts or entertaining BlaBlaCar drivers) [14], [26], [27].

Research has thus focused on the role of social experience and benefits beyond economic and product-related considerations for platform usage. In fact, social motives are frequently reported as a driver of consumption on C2C platforms [28]-[30]. Moreover, consumers' intention to use said platforms is shown to be positively influenced by social utility [31]-[34]. In consequence, providers can benefit from users' expectations of social value and accordingly market their product and themselves as a centerpiece of the overall usage experience [35], [36]. Thus, the prospect of interpersonal trust from social contact enables these providers to become their own brand [8]. However, this works only, if the transacted product or service is related to personal interaction. If the interaction is limited to the exchange of standardized goods on an e-commerce platform, for example, there is little need in providing "brand-building" trust cues.

In cases where the degree of social interaction is high, platforms provide dedicated means to create expressive user profiles. Trust towards a prospective interaction partner, therefore, hinges on what is conveyed through the platforms' user interfacesincluding supposedly incidental clues such as personal 
preferences, facial expressions, wording, etc. Previous findings suggest that high-quality sellers display a wider array of signals [37] and recent research has begun to examine this particular role of user representation explicitly [10], [38]-[40]. Moreover, the proliferation of trust mechanisms has raised interest in view of regulation as there arise new potential harms, including new forms of discrimination [41] as well as possibilities for strategic manipulation and market failure [42].

On a different note, a user's verified identityvalidated and vouched for by the platform or an external institution-can signal authenticity to other participants and platform operators have developed a range of mechanisms [8], [43]. Such identity verification was shown to increase trust, for instance in online dating [44]. In the context of peer-to-peer accommodation sharing (e.g., Airbnb), the availability of verified identity is a popular research topic [45][47]. While some studies state a positive effect of verified identity on prices [48] others find no significant effects [23], [49]. Another means of identity verification is the practice of linking social network accounts across online platforms [50], [51].

Moreover, platforms deploy additional mechanisms to promote trust in service providers. The use of badges as a sign of achievement has been described in the context of social networks, but can generally be extended to all types of digital platforms [52]. Empirical analyses show the effects of these badges on Airbnb ("superhosts"; [23], [49], [53], [54]) but usage is prevalent on other platforms as well (e.g., Uber; [55]). Further implicit information includes the number of transactions/reviews and users' membership duration [22], [23], [49].

Beyond mechanisms to increase trust (e.g., through ratings), platforms also employ means to mitigate risks (e.g., fraud) and hence reduce users' trust thresholds. Such "e-commerce institutional mechanisms" include insurances and warranties, escrow payment services, and privacy assurances [56]. In addition to trust into prospective transaction partners, also trust into the platform itself represents a prerequisite for transactions to materialize. Importantly, a platform's trustworthiness is suggested to rub off on the providers on the platform ("trust transfer"; [57]-[59]).

While extant research has mainly considered platforms and trust cues in isolation, with this paper, we extend this body of literature by providing a cross-platform trust cue "panorama" and classification, which allows to take the varying degrees of social interaction as a driver of the usage and adequacy of trust cues into account.

\section{How platforms build trust}

\subsection{Taxonomy for trust-building cues}

Overall, platforms use a variety of mechanisms for reputation management and trust-building. Based on our review of related work and in situ exploration across a broad range of platforms from different contexts, we propose the following categorization:

(1) Transaction-based trust cues refer to evaluations provided by prior transaction partners. These may be numeric (e.g., star rating scales, positive/negative) or written assessments (e.g., text reviews).

(2) Socially rich and Expressive User Profiles are created and organized by users individually. Typical content includes photos/profile images, self-descriptions, video messages, or references to external resources. We hypothesize that such trust cues' importance increases with a platform's degree of social interaction.

(3) Next, Identity Verification refers to the platform examining a user's personal identity and authenticity, for instance, by email or phone number confirmation. Moreover, depending on context, identity verification may include the provision of an ID card, licenses, or linkage to online social network accounts.

(4) Last, Implicit Information and distinctions such as status badges or the display of a users' membership duration, number of transactions, or average response time are provided by the platform and accentuate a user's particular status, behaviors, achievements, or merits.

\subsection{Degree of Social Interaction (DoSI)}

Table 1 introduces the notion of platforms' degree of social interaction-defined by the level of personal interaction and social experiences on a given platform [25]. A platform's classification is based on two factors: (1) the necessity for and degree of physical encounters for service provision and (2) the extent to which personal interaction with the service provider is part of the overall value proposition - in addition (and as opposed to) focusing solely on the underlying product or service. Hence, where personal experience becomes a constituent part of the overall value proposition, Expressive User Profiles are particularly well-suited to convey information to prospective consumers. One would expect this to be reflected in 
Table 1. Degree of Social Interaction (DoSI)

\begin{tabular}{|c|l|} 
DoSI & Rationale \\
Low & $\begin{array}{l}\text { No physical encounters between seller and buyer; } \\
\text { very limited online communication; focus entirely on } \\
\text { the sold product; interaction purely transactional } \\
\text { (e.g., e-commerce) }\end{array}$ \\
Moderate & $\begin{array}{l}\text { Physical encounter with provider possible (e.g., brief } \\
\text { interaction for service handover); focus on underlying } \\
\text { commodity (e.g., apartment, car); personal interaction } \\
\text { may add to the value proposition } \\
\text { (e.g., accommodation, carsharing) }\end{array}$ \\
\hline High & $\begin{array}{l}\text { Physical encounter with provider prerequisite to } \\
\text { service provision; consumers entrust physical safety, } \\
\text { access to property to provider; focus on service and } \\
\text { provider; personal interaction part of value proposition } \\
\text { (e.g., accommodation, mobility, crowd work) }\end{array}$ \\
\hline $\begin{array}{l}\text { Note: Within in the same type of platform, nuances may occur for the DosI and are } \\
\text { reflected here (e.g., staying in a shared apartment (high Dosl) vs. renting an entire } \\
\text { home (moderate DosI) on an accommodation platform) }\end{array}$ \\
\hline
\end{tabular}

both the availability of expressive trust cues as well as their perceived importance.

Proposition: A platform's degree of social interaction is positively related to (1) the usage of Expressive User Profile elements by providers and (2) the importance of such cues in the eyes of consumers.

\section{Methods}

Data Collection 1: To examine how platforms build trust (empirically) and to evaluate our proposition, we draw on actual and recent market data. In a first step, we inspect the outlined platforms and trust-building mechanisms manually. Note that within the scope of this paper, we focus on trust into the providers (i.e., hosts, drivers, workers, lessors, sellers). Naturally, providers' trust into consumers is essential as well since providers face considerable levels of economic exposure and, after all, usually have the last say in whether a transaction will actually be realized. Using web scraping (implemented in Java), we then retrieve samples of user profiles and the associated data such as ratings, descriptions, profile images, and so forth for each platform. Overall, we queried 11 platforms collecting data points of more than 42,000 user profiles. Data was collected between August and September 2018 and is available from the authors upon request. Table 2 summarizes the web-crawling results on the use and characteristics of different trust cues on the respective platforms (Airbnb, Homestay, Wimdu, EasyCarClub, Drivy, Turo, BlaBlaCar, Wingly, eBay, Gumtree, TaskRabbit).

Data Collection 2: As a second step, we launched an online survey. Participants were recruited via Prolific.ac [60] with a total of 204 participants from across the EU, the US, Canada, and Israel. We controlled for online shopping frequency (i.e., more than once per month) and age (i.e., 18-49; mean=33.7, $66 \%$ female) and equally divided study participants among five platform types (accommodation, car, mobility, e-commerce, crowd work). A total of 187 valid responses remained after attention and completeness checks. Based on the empirical findings, we asked participants to rate the overall importance of the different trust-building mechanisms as well as the importance of individual artifacts and properties (such as face visibility) on 7-point Likert scales. We added open-ended questions that were manually coded into categories, for example, other drivers of trust and distrust, and general drivers of platform use. The data was collected in May 2019, reviewed, and coded independently by two researchers. Table 3 summarizes the survey results on the importance of specific trustbuilding mechanisms per platform type.

Table 2. Platforms' and users' use of trust-building mechanisms (based on Data Collection 1)

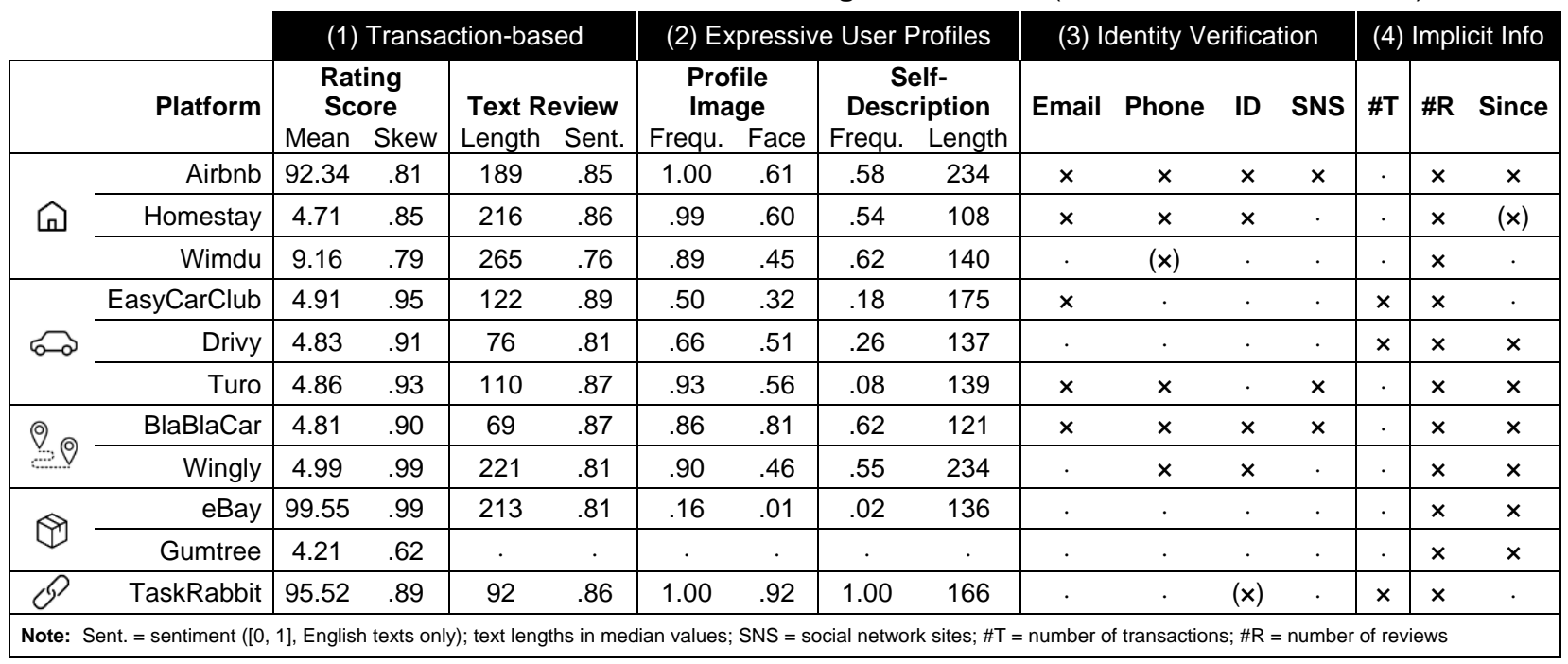




\section{Results}

\subsection{Platform \& Provider Perspective}

Table 2 summarizes the platforms' use of trust-building mechanisms and how these are being utilized by their users. As can be seen, many of the identified artefacts such as rating scores, text reviews, and profile images are present on most platforms, while others are less common (e.g., social media linkage). Moreover, there are marked and platformcontingent differences in how they are being used.

Rating Scores-All considered platforms use some sort of numerical rating score. The mechanisms vary in terms of scales, aggregation, granularity, and display. While some platforms display a five-star rating scale rounded to half stars (e.g., Airbnb, Turo), other platforms offer a more fine-grained display (e.g., .10 stars; BlaBlaCar, Gumtree). We observe rather skewed rating score distributions where a majority of users on most platforms exhibit highly positive ratings. This is not unexpected since rating scale skewness represents a common phenomenon across platforms and domains [61], [62]. Figure 1 shows the cumulative (log-scaled) fraction of users with ratings smaller or equal to a specific rating score (from $\min$ to max). Most platforms exhibit rather similar patterns of rating score skewness, while there exist outliers in both directions. Table 2 reports a quantification of the platforms' rating score skewness (determined similar to the Gini index with $0 \leq$ skewness $\leq 1$ ). For a recent study on the antecedents of such extreme distributions on review sites and platforms, we refer to [62].

Profile Images-Faces create trust [10], [63]. It is hence not surprising that almost all platforms offer the possibility to upload a profile photo. Note, however, that there occur marked differences in how this option

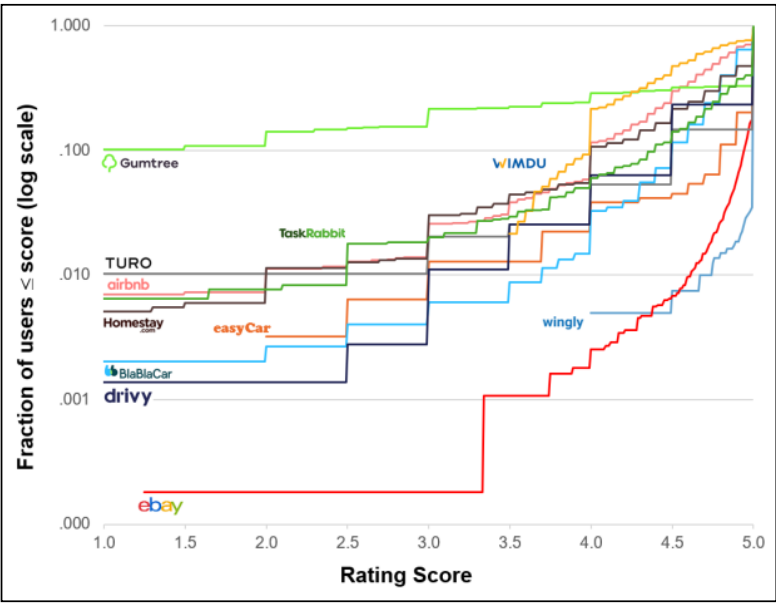

Figure 1. Rating distribution across platforms is exerted by the users. For instance, users may upload a profile photo both on eBay and Airbnb but, while virtually all hosts on Airbnb have uploaded a photo (99.8\%), only a minority has done so on eBay (15.8\%); a finding that is in line with our proposition. In addition, facial recognition analysis (using Microsoft's Cognitive Services [64]) reveals that the fraction of users with a "proper" profile photo, that is, a photo for which a face is detected by the software, ranges from $1 \%$ (eBay) to $92 \%$ (TaskRabbit).

Text-Moreover, the way people write about themselves and others differs between platforms (e.g., in terms of text length and sentiment). While on eBay, only $2 \%$ of sellers provide a (rather short) personal description about themselves, the saliency of textual self-descriptions is significantly higher on platforms characterized by a higher degree of social interaction (e.g., Airbnb: 58\%). Similarly, reviews vary considerably in length between platforms. Also, there exist subtle platform-specific differences of how users write about each other. Text sentiment analysis [65] shows that a vast majority of text reviews is highly positive (ranging from 0 to 1 ).

Identity Verification-Mechanisms for identity verification are less widely used across platforms. While Airbnb and BlaBlaCar display a wide range of identity verifications (email, phone, id, social network sites), other platforms (e.g., eBay, Drivy) do not make any use of it.

User Activity-Another property for platform distinction roots in the concentration of user activity as indicated by the number of completed (and reviewed) transactions. Figure 2 shows the cumulative fractions of user activity against the user samples. As can be seen, concentration varies markedly.

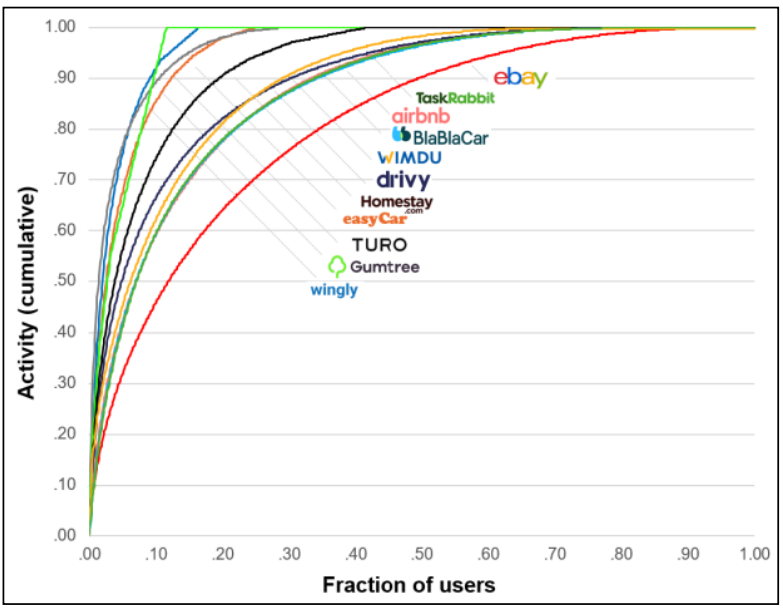

Figure 2. Activity concentration across platforms 
Table 3. Importance of trust-building mechanisms per platform type

\section{\begin{tabular}{l|l|l} 
(1) Transaction-based & (2) Expressive User Profiles & (3) Identity Verification
\end{tabular}}

(4) Implicit Info

\begin{tabular}{|c|c|c|c|c|c|c|c|c|c|c|c|c|}
\hline & Platform type & $\begin{array}{l}\text { Rating } \\
\text { Score }\end{array}$ & $\begin{array}{c}\text { Text } \\
\text { Review }\end{array}$ & $\begin{array}{l}\text { Profil } \\
\text { Imp. }\end{array}$ & $\begin{array}{c}\text { Image } \\
\text { Face }\end{array}$ & $\begin{array}{c}\text { Self- } \\
\text { Description }\end{array}$ & Email & Phone & ID & SNS & \#T & Since \\
\hline$\widehat{n}$ & Accommodation & 6.59 & 6.36 & 5.05 & 4.49 & 3.69 & 6.49 & 6.44 & 6.56 & 4.41 & 6.15 & 5.85 \\
\hline డ- & Carsharing & 6.31 & 5.94 & 4.39 & 4.44 & 3.83 & 5.89 & 6.31 & 6.53 & 3.64 & 5.75 & 5.69 \\
\hline$\theta$ & Mobility & 6.22 & 5.41 & 6.16 & 6.43 & 2.89 & 5.32 & 6.14 & 6.70 & 3.03 & 5.59 & 5.54 \\
\hline (9) & E-commerce & 6.15 & 5.85 & 2.98 & 2.40 & 2.18 & 5.53 & 4.93 & 5.18 & 3.43 & 5.85 & 5.03 \\
\hline 8 & Crowd work & 6.11 & 5.89 & 4.63 & 4.86 & 4.69 & 5.86 & 5.94 & 6.00 & 3.89 & 5.57 & 5.23 \\
\hline
\end{tabular}

Note: $\mathrm{n}=187$; values on Likert scale $[1,7] ; \mathrm{Imp} .=$ importance; $\mathrm{SNS}=$ social network sites; \#T = number of transactions; $\mathrm{x}=$ importance $>6.0 ; \mathrm{x}=$ importance $<4.0$

\subsection{Consumer Perspective}

To better understand platform users' preferences, we asked survey participants to rate how important they personally deem the different trust cues across platform contexts (Table 3; 7-point Likert scales labelled as (1)="not important at all" to (7)="very important"). Several observations are remarkable:

Rating scores-Within transaction-based trust cues, rating scores unanimously score highest in importance when deciding to book/buy a service from a prospective provider (i.e., consistently rated above 6 out of 7). This appears natural given ratings represent the most commonly deployed signal. Users rely on it more than on text reviews in each investigated platform type though the latter also receives generally high ratings for importance.

Figure 3 depicts both actually observed rating scores from across the 11 platforms and the category-specific trust thresholds. These thresholds are based on survey respondents' stated star rating score (on a scale of 1.0-5.0 stars) above which they would generally consider a provider as trustworthy. All platform rating scores have been normalized to the 1-5 stars interval accordingly. A striking observation is that the average thresholds are much lower than the vast majority of actual ratings with only very few outliers falling short of the thresholds.

Profile images-We observe marked differences in the perceived importance of profile images. The results seem to be mirroring our market data findings and corroborate our reasoning on the degree of social

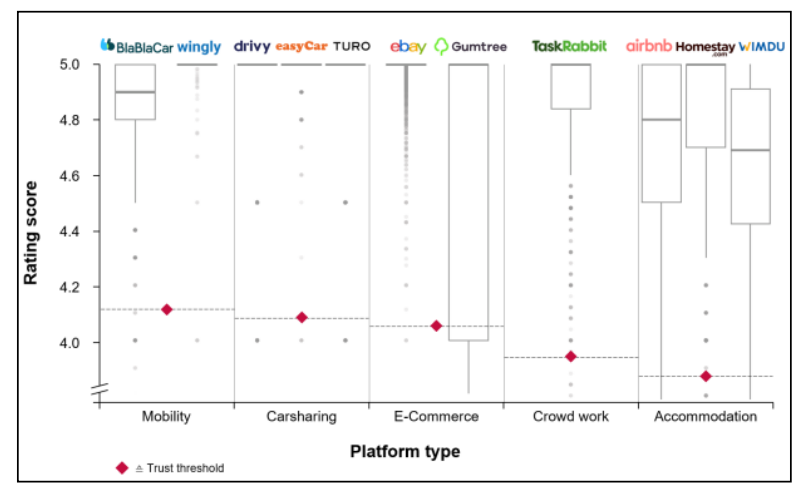

Figure 3. Actual scores vs. trust thresholds interaction. Especially low ratings within the ecommerce category (2.98) are well in line with actual usage of profile images on these platforms (only 16\% and no option to upload, respectively); if users do not find it important, as their interaction with transaction partners is limited, then there is not much reason to upload one. However, there is a high demand for profile images on mobility platforms and visible faces (each >6). As we have seen, not all providers use profile images on the investigated platforms but may benefit from doing so.

Self-description-Participants were explicitly asked to rate the availability of self-descriptive elements such as hobbies, occupation, or personal background. Overall, these seem to play a minor role for building trust. Only in the crowd work category did participants rated them as important. When prompted to comment on particularly helpful pieces of personal information "skills" and "experience from previous jobs" occurred frequently. Other than that, users seem to be somewhat indifferent towards extensive use of such trust cues.

Identity verification -We notice clear support for verified provider identity, especially on platforms with the potential for higher degrees of social and in-person interaction such as mobility, crowd work, and accommodation but also for carsharing. In e-commerce these elements play a less important role. This makes sense given its rather transactional-focused and impersonal nature. It is noteworthy, however, that the linkage of social network accounts is not deemed as of high importance.

When directly asked for additional trust cues users would like to see, the provider's age came in on top with $11 \%$ of all participants mentioning this aspect explicitly (17\% and $14 \%$ for carsharing and mobility, respectively). Albeit demanding this piece of information can be considered discriminatory, long years of experience and a certain maturity seem to play a role for services that involve driving or renting out vehicles. Other elements that have received mentions are legal background checks for providers on mobility $(27 \%)$ and accommodation platforms (5\%). Detailed policies on this topic are opaque though at least some platforms seem to be conducting background checks in some jurisdictions (e.g., Airbnb, Uber). 


\subsection{DoSI Perspective}

To evaluate our proposition, Figure 4 combines both platform and user perspective. It plots the empirically observed frequency of trust cues from the Expressive User Profiles category (i.e., profile image availability, face visibility, self-description availability; Table 2) for the 11 platforms against the stated importance of these elements by survey participants per platform type (Table 3; $\mathrm{n}=33$ ). Drawing on our reasoning from Table 1, the color-coding represents the platform's respective degree of social interaction ${ }^{1}$. This value seems to explain both frequency and importance of "soft" trust cues well. In addition, the fitted regression indicates a positive relation between actual usage of these mechanism and perceived importance (Figure 4).

First, usage frequency and importance are in fact correlated positively (Pearson $r=.711, \quad p<.001$ ). Moreover, two OLS regression analyses provide support for the conjectured relations between a platform's degree of social interaction (DoSI; coded numerically as 1 to 3 ) and providers' use of different trust cues on the platform $\left(b=.362, p<.001 ; \mathrm{R}^{2}=.53\right.$; $\mathrm{n}=33$ ) as well as between platform domain's DoSI and consumers' assessment of the importance of trust cues within that domain $\left(b=1.129, p<.01 ; \mathrm{R}^{2}=.50 ; \mathrm{n}=15\right)$.

We acknowledge the fact that nuances in the DoSI may occur depending on the type of transaction within a particular platform (see Table 1 for an explanation). Hence, we varied the DoSI value for accommodation platforms as a sensitivity check for our model. For instance, alternating the DoSI score from "moderate" to "high" for accommodation sharing yielded almost identical estimates for the regressions.

\section{Discussion and Conclusion}

The rise of the platform economy has led to the emergence of multi-sided markets "that shape the terms on which participants interact with one another" [3]. Given the paramount role of platforms' design choices for many of today's social and economic interactions, this paper sets out to provide a practical overview and empirical insight into the ways platforms build trust between their users and, in turn, how those measures are perceived by platform participants.

As outlined, for many of today's platforms dealing with experience products, a blurring occurs between trust-building, marketing, and product/service descriptions. Beyond the role for trust-building, personal information about providers can play a

\footnotetext{
${ }^{1}$ Low: e-commerce; Medium: carsharing, accommodation; High: mobility, crowd work
}

substantial role in the offer's value proposition as many people explicitly look for social value such as authenticity, good conversations and company, or cultural experiences [14], [27]. In this sense, providers on peer-to-peer platforms have themselves become part of the product they are offering and will make use of the full breadth of the trust cue palette to advertise their services. Our analysis yields some key take-aways along those lines:

Most of the studied platforms employ all four basic types of trust-building mechanisms as introduced within this work (Transaction-based cues, Expressive User Profiles, Identity Verification, Implicit Information). Despite several similarities, there exist marked differences in how these mechanisms are being utilized and how their importance is perceived by the platforms' users. Specifically, user profiles are particular expressive on platforms that are characterized by a high degree of social interaction. This is in line with the finding that "social aspects and individual characteristics become more relevant in this particular context" $[25$, p. 26]. Contrary, expressiveness is much lower on platforms for renting and selling, i.e. those platforms on which user interactions are limited. This justifies the labeling of elements from the Expressive User Profiles category as soft trust cues - when deciding to book or buy from a particular provider, users, in many cases, deem "hard" trust cues (especially Transaction-based cues and Identity Verification but also Implicit Information) of higher importance.

Now, when accepting the premise that there exists a link between a platform's degree of social interaction and its use of expressive trust cues, one may draw on any platform's use of such cues to infer its degree of social interaction, providing a convenient and efficient parameter to compare platforms. It strikes the eye that especially e-commerce platforms such as Gumtree and eBay (and their users) do not make use of the full breadth of user profile design and identity verification. While it appears plausible that the rather anonymous

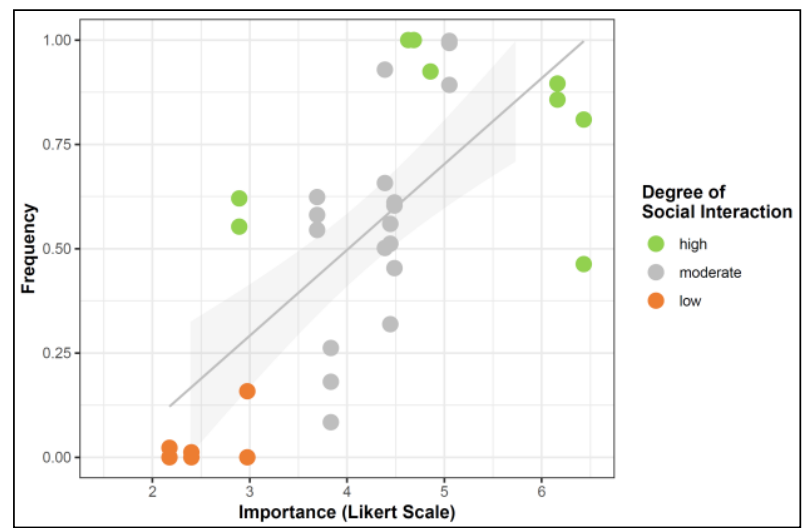

Figure 4. Frequency of social cues vs. importance 
and impersonal nature of these platforms' transactions does in fact not necessitate the use of such means, it seems natural to ask whether they may nevertheless benefit from doing so.

Limitations \& Future Work-Our approach does not come without its caveats and limitations and we delegate it to future work to explore the causes, effects, mechanisms, and limits of trust-building within the platform landscape in greater detail. A first suggestion is to extend our assessment to analyze the trustbuilding categories beyond Expressive User Profiles to uncover platform (type-) specific drivers of their usage in-depth. Further, the focus of this study could be broadened to encompass trust into consumers, that is, the opposite market side. Also, the rapid emergence and vanishing of platform-based businesses will yield new players, trust requirements, and mechanisms, all of which future research will have to take into account. One technical limitation roots in the use of Microsoft's cognitive services for text sentiment analysis and facial recognition. The latter appears to be rather conservative in flagging faces, resulting in many false negatives. Thus, the estimates for face visibility in Table 2 should be seen as lower benchmarks. Future work may want to apply more sophisticated tools and methods to our data.

On average, $51 \%$ of survey participants had used one or more platform from their assigned segment (i.e., accommodation, carsharing, mobility, e-commerce, crowd work). Yet, there occurred considerable category-specific differences. While 93\% of respondents had previously used e-commerce platforms, only $14 \%$ stated so for carsharing. Hence, these results must be interpreted with some caution as a considerable fraction of respondents in some categories does not have actual insider experience. Future research may want to draw upon more experienced subjects. Moreover, there exist other ways of trust formation beyond the mechanisms considered here. Examples include pre-purchase communication such as typical for Taobao [66] or the formation of social capital through repeated interactions [67].

Eventually, in view of the multiplicity of platforms and the many parallel, unconnected reputation silos ("isolated islands"; [8]), recent research has set out to consider the transfer of reputation between platforms [9], [24], [68]. While platforms could thus build trust by allowing their users to import reputation from other platforms, none of the considered platforms is currently offering such a functionality. Neither are third-party solutions to reputation portability (as of yet) well known [58]. In fact, when asked about additional desirable trust cues, only one participant explicitly mentioned the use of an "identity verifying or reputation service". However, a lot of people would value imported reputation as, on average, the concept scored 5.7 on the 7-point importance scale. High-DoSI platforms (i.e., crowd work, mobility) exhibited strongest desirability of the feature and text reviews imported from other platforms were appreciated slightly more than imported star ratings [69].

Conclusion-Trust and reputation among users of two-sided platforms are imperative for flourishing markets. The process of building and maintaining trust is hence of utmost importance to platforms. As we have shown, given the specific nature of a platform type, the choice of adequate means is contextual. For instance, nuances in the degree of social interactions on platforms impact both the provision of trust cues by platforms as well as the perceived importance in the eyes of their users. Our results with regard to the close relation between the degree of social interaction and the importance of trust-building mechanisms may be instructive for platform managers and related business models that involve the handling of online trust and reputation: the more a platform's value proposition hinges on social interactions, the more important it is to provide expressive trust cues. By providing an empirical basis and first cross-platform insights into the use of reputation systems and trust-building mechanisms (both by platforms and users), we hope to contribute to making the discussion more explicit, if not even more objective.

\section{References}

[1] M. Van Alstyne, G. Parker, and S. P. Choudary, "Pipelines, platforms, and the new rules of strategy," Harv. Bus. Rev., vol. 94, no. 4, pp. 54-63, 2016.

[2] C. Stummer, D. Kundisch, and R. Decker, "Platform launch strategies," Bus. Inf. Syst. Eng., vol. 60, no. 2, pp. 167-173, 2018.

[3] M. Kenney and J. Zysman, "The rise of the platform economy," Issues Sci. Technol., vol. 32, no. 3, 2016.

[4] T. Economist, "The rise of the sharing economy," Economist, 2013. [Online]. Available: https://www.economist.com/leaders/2013/03/09/therise-of-the-sharing-economy. [Accessed: 14-May2019].

[5] A. Sundararajan, The Sharing Economy: The End of Employment and the Rise of Crowd-Based Capitalism. The MIT Press, 2016.

[6] T. Teubner, "The web of host-guest connections on Airbnb: a network perspective," J. Syst. Inf. Technol., vol. 20, no. 3, pp. 262-277, 2018.

[7] S. Tadelis, "Reputation and Feedback Systems in Online Platform Markets," Annu. Rev. Econom., vol. 8, no. 1, pp. 321-340, Oct. 2016.

[8] F. Mazzella, A. Sundararajan, V. Butt d'Espous, and M. Möhlmann, "How Digital Trust Powers the Sharing Economy: The Digitization of Trust," IESE Insight, no. 30, pp. 24-31, 2016. 
[9] M. Hesse and T. Teubner, "Reputation Portability Quo Vadis?," Electronic Markets. (in press), 2019.

[10] E. Ert, A. Fleischer, and N. Magen, "Trust and reputation in the sharing economy: The role of personal photos in Airbnb," Tour. Manag., vol. 55, pp. 62-73, Aug. 2016.

[11] T. Teubner and D. Dann, "How Platforms Build Trust," SSRN Electron. J., 2018.

[12] M. Granovetter, "Economic Action and Social Structure: The Problem of Embeddedness," Am. J. Sociol., vol. 91, no. 3, pp. 481-510, Nov. 1985.

[13] M. Granovetter, "The Impact of Social Structure on Economic Outcomes," J. Econ. Perspect., vol. 19, no. 1, pp. 33-50, Feb. 2005.

[14] F. Hawlitschek, T. Teubner, and H. Gimpel, "Consumer motives for peer-to-peer sharing," J. Clean. Prod., vol. 204, pp. 144-157, 2018.

[15] A. Zarifis, X. Cheng, and J. Kroenung, "Collaborative consumption for low and high trust requiring business models: from fare sharing to supporting the elderly and people with disability," Int. J. Electron. Bus., vol. 15, no. $1,2019$.

[16] F. Hawlitschek, T. Teubner, and C. Weinhardt, "Trust in the Sharing Economy," Die Unternehmung, vol. 70, no. 1, pp. 26-44, 2016.

[17] M. Möhlmann, "Collaborative consumption: determinants of satisfaction and the likelihood of using a sharing economy option again," J. Consum. Behav., vol. 14, no. 3, pp. 193-207, 2015.

[18] P. Resnick, R. Zeckhauser, E. Friedman, and K. Kuwabara, "Reputation systems," Commun. ACM, vol. 43, no. 12, pp. 45-48, Dec. 2000.

[19] G. Bolton, B. Greiner, and A. Ockenfels, "Engineering Trust: Reciprocity in the Production of Reputation Information," Manage. Sci., vol. 59, no. 2, pp. 265-285, Feb. 2013.

[20] S. Ba and P. A. Pavlou, "Evidence of the Effect of Trust Building Technology in Electronic Markets: Price Premiums and Buyer Behavior," MIS Q., vol. 26, no. 3, pp. 243-268, Sep. 2002.

[21] P. Resnick, R. Zeckhauser, J. Swanson, and K. Lockwood, "The value of reputation on eBay: A controlled experiment," Exp. Econ., vol. 9, no. 2, pp. 79-101, 2006.

[22] W. Qiu, P. Parigi, and B. Abrahao, "More Stars or More Reviews? Differential Effects of Reputation on Trust in the Sharing Economy," 2018 CHI Conf. Hum. Factors Comput. Syst., 2018.

[23] T. Teubner, F. Hawlitschek, and D. Dann, "Price Determinants on Airbnb: How Reputation Pays Off in the Sharing Economy," J. Self-Governance Manag. Econ., vol. 5, no. 4, p. 53, 2017.

[24] L. Otto, P. Angerer, and S. Zimmermann, "Incorporating external trust signals on service sharing platforms," in ECIS 2018 Proceedings, 2018.

[25] M. Möhlmann, "Digital Trust and Peer-to-Peer Collaborative Consumption Platforms: A Mediation Analysis," SSRN Electron. J., 2016.

[26] D. Dann, M. H. Duong, E. Hoeven, T. Teubner, and C. Weinhardt, "How Airbnb conveys social and economic value through user representation," in Proceedings of the Workshop on Designing User Assistance in Interactive Intelligent Systems, 2018, pp. 32-43.

[27] I. P. Tussyadiah, "Factors of satisfaction and intention to use peer-to-peer accommodation," Int. J. Hosp. Manag., vol. 55, pp. 70-80, 2016.

[28] R. Botsman and R. Rogers, What's Mine Is Yours: The Rise of Collaborative Consumption. Harper Business, 2010.

[29] I. P. Tussyadiah, "An Exploratory Study on Drivers and Deterrents of Collaborative Consumption in Travel," in Information and Communication Technologies in Tourism 2015, Cham: Springer International Publishing, 2015, pp. 817-830.

[30] V. Bellotti, A. Ambard, D. Turner, C. Gossmann, K. Demkova, and J. M. Carroll, "A Muddle of Models of Motivation for Using Peer-to-Peer Economy Systems," in Proceedings of the 33rd Annual ACM Conference on Human Factors in Computing Systems - CHI '15, 2015, pp. 1085-1094.

[31] D. Schaffner, D. Georgi, and E. Federspiel, "Comparing Customer Experiences and Usage Intentions Regarding Peer-to-Peer Sharing Platforms with Conventional Online Booking Websites: The Role of Social, Hedonic, and Functional Values," P. Rossi, Ed. Cham: Springer International Publishing, 2017, pp. 1049-1056.

[32] A. Oyedele and P. Simpson, "Emerging adulthood, sharing utilities and intention to use sharing services," $J$. Serv. Mark., vol. 32, no. 2, pp. 161-174, Apr. 2018.

[33] S. J. Barnes and J. Mattsson, "Understanding collaborative consumption: Test of a theoretical model," Technol. Forecast. Soc. Change, vol. 118, pp. 281-292, May 2017.

[34] E. Bucher, C. Fieseler, and C. Lutz, "What's mine is yours (for a nominal fee) - Exploring the spectrum of utilitarian to altruistic motives for Internet-mediated sharing," Comput. Human Behav., vol. 62, pp. 316-326, Sep. 2016.

[35] I. P. Tussyadiah, "Strategic Self-presentation in the Sharing Economy: Implications for Host Branding," in Information and Communication Technologies in Tourism 2016, vol. 1, A. Inversini and R. Schegg, Eds. Cham: Springer International Publishing, 2016, pp. 695-708.

[36] L. Harris and A. Rae, "Building a personal brand through social networking," J. Bus. Strategy, vol. 32, no. 5, pp. 14-21, 2011.

[37] T. Mavlanova, R. Benbunan-Fich, and M. Koufaris, "Signaling theory and information asymmetry in online commerce," Inf. Manag., vol. 49, no. 5, pp. 240-247, 2012.

[38] O. Abramova, H. Krasnova, and C.-W. Tan, "How much will you pay? Understanding the value of information cues in the sharing economy," in ECIS 2017 Proceedings, 2017, pp. 1-18.

[39] A. Fagerstrøm, S. Pawar, V. Sigurdsson, G. R. Foxall, and M. Yani-de-Soriano, "That personal profile image might jeopardize your rental opportunity! On the relative impact of the seller's facial expressions upon buying behavior on Airbnb ${ }^{\mathrm{TM}}$," Comput. Human Behav., vol. 72, pp. 123-131, 2017.

[40] X. Ma, J. T. Hancock, K. L. Mingjie, and M. Naaman, 
"Self-disclosure and perceived trustworthiness of Airbnb host profiles," in CSCW 2017 Proceedings, 2017, pp. 1-13.

[41] B. G. Edelman, M. Luca, and D. Svirsky, "Racial discrimination in the sharing economy: Evidence from a field experiment," Am. Econ. J. Appl. Econ., vol. 9, no. 2, pp. 1-22, 2017.

[42] M. Watt and H. Wu, "Trust mechanisms and online platforms: A regulatory response," 2018.

[43] A. Sundararajan, "Peer to peer business and the sharing (collaborative) economy: Overview, Economic Effects and Regulatory Issues," 2014, pp. 1-7.

[44] G. Norcie, E. De Cristofaro, and V. Bellotti, "Bootstrapping trust in online dating: Social verification of online dating profiles," Lect. Notes Comput. Sci., vol. 7862 LNCS, pp. 149-163, 2013.

[45] D. Guttentag, "Airbnb: disruptive innovation and the rise of an informal tourism accommodation sector," Curr. Issues Tour., vol. 18, no. 12, pp. 1192-1217, 2015.

[46] S. Dolnicar, Peer-to-Peer Accommodation Networks. Goodfellow Publishers, 2017.

[47] J. Jung and K.-P. Lee, "Curiosity or Certainty? A Qualitative , Comparative Analysis of Couchsurfing and Airbnb User Behaviors," in Proceedings of the 2017 CHI Conference Extended Abstracts on Human Factors in Computing Systems - CHI EA '17, 2017, pp. 17401747.

[48] D. Wang and J. L. Nicolau, "Price determinants of sharing economy based accommodation rental: A study of listings from 33 cities on Airbnb.com," Int. J. Hosp. Manag., vol. 62, pp. 120-131, 2017.

[49] K. Xie and Z. Mao, "The impacts of quality and quantity attributes of Airbnb hosts on listing performance," Int. J. Contemp. Hosp. Manag., vol. 29, no. 9, pp. 2240-2260, Sep. 2017.

[50] Y. Chen, C. Zhuang, Q. Cao, and P. Hui, "Understanding Cross-site Linking in Online Social Networks," in Proceedings of the 8th Workshop on Social Network Mining and Analysis - SNAKDD'14, 2014, vol. 12, no. 4, pp. 1-9.

[51] G. Silvestri, J. Yang, A. Bozzon, and A. Tagarelli, "Linking accounts across social networks: The case of StackOverflow, Github and Twitter," CEUR Workshop Proc., vol. 1489, pp. 41-52, 2015.

[52] K. H. Kwon, A. Halavais, and S. Havener, "Tweeting Badges: User Motivations for Displaying Achievement in Publicly Networked Environments," Cyberpsychology, Behav. Soc. Netw., vol. 18, no. 2, pp. 93-100, Feb. 2015.

[53] J. Neumann and D. Gutt, “A homeowner's guide to airbnb: Theory and empirical evidence for optimal pricing conditional on online ratings," in ECIS 2017 Proceedings, 2017, vol. 2017, pp. 997-1010.

[54] E. Ert and A. Fleischer, "The evolution of trust in Airbnb: A case of home rental," Ann. Tour. Res., vol. 75, no. November 2018, pp. 279-287, 2019.
[55] N. Scheiber, "How Uber Uses Psychological Tricks to Push Its Drivers' Buttons - The New York Times," The New York Times, 2017. [Online]. Available: https://www.nytimes.com/interactive/2017/04/02/techno logy/uber-drivers-psychological-tricks.html?emc=eta1 . [Accessed: 14-May-2019].

[56] Y. Fang, I. Qureshi, H. Sun, P. McCole, E. Ramsey, and K. H. Lim, "Trust, satisfaction, and online repurchase intention: The moderating role of perceived effectiveness of e-commerce institutional mechanisms," MIS Q., vol. 38, no. 2, pp. 407-427, 2014.

[57] X. Chen, Q. Huang, R. M. Davison, and Z. Hua, "What drives trust transfer? The moderating roles of sellerspecific and general institutional mechanisms," Int. J. Electron. Commer., vol. 20, no. 2, pp. 261-289, 2015.

[58] T. Teubner, F. Hawlitschek, and M. T. P. Adam, "Reputation Transfer," Bus. Inf. Syst. Eng., vol. 61, no. 2, pp. 229-235, 2019.

[59] M. Möhlmann and A. Geissinger, "Trust in the Sharing Economy: Platform-Mediated Peer Trust," in The Cambridge Handbook of the Law of the Sharing Economy, vol. 70, no. 1, 2018, pp. 26-44.

[60] S. Palan and C. Schitter, "Prolific.ac-A subject pool for online experiments," J. Behav. Exp. Financ., vol. 17, pp. 22-27, 2018.

[61] G. Zervas, D. Proserpio, and J. Byers, "A First Look at Online Reputation on Airbnb, Where Every Stay is Above Average," SSRN Electron. J., pp. 1-22, 2015.

[62] V. Schoenmueller, O. Netzer, and F. Stahl, "The Extreme Distribution of Online Reviews: Prevalence, Drivers and Implications," SSRN Electron. J., 2018.

[63] T. Teubner, M. T. P. Adam, S. Camacho, and K. Hassanein, "Understanding resource sharing in $\mathrm{C} 2 \mathrm{C}$ platforms: The role of picture humanization," in ACIS 2014 Proceedings, 2014, pp. 1-10.

[64] Microsoft, "Cognitive Services » Face.” 2018.

[65] Microsoft, "How to detect sentiment in text analytics," 2018. [Online]. Available: https://docs.microsoft.com/en-us/azure/cognitiveservices/text-analytics/how-tos/text-analytics-how-tosentiment-analysis.

[66] Q. Huang, X. Chen, C. X. Ou, R. M. Davison, and Z. Hua, "Understanding buyers' loyalty to a C2C platform: The roles of social capital, satisfaction and perceived effectiveness of e-commerce institutional mechanisms," Inf. Syst. J., vol. 27, no. 1, pp. 91-119, 2017.

[67] X. Chen, Q. Huang, and R. M. Davison, "Economic and social satisfaction of buyers on consumer-to-consumer platforms: The role of relational capital," Int. J. Electron. Commer., vol. 21, no. 2, pp. 219-248, 2017.

[68] T. Teubner, M. T. P. Adam, and F. Hawlitschek, "Unlocking online reputation: On the effectiveness of cross-platform signaling," Bus. Inf. Syst. Eng., vol. (in press), 2019.

[69] M. Hesse and T. Teubner, "Takeaway Trust: A market data perspective on reputation portability in electronic commerce," HICSS 2020 Proceedings. (in press), 2020. 